\title{
A Fast Readout Electronic System for Accurate Spatial Detection in Ion Beam Tracking for the Next Generation of Particle Accelerators
}

\author{
Alejandro Garzón-Camacho, Begoña Fernández, Marcos A. G. Alvarez, \\ Joaquín Ceballos, and José M. de la Rosa, Senior Member, IEEE
}

\begin{abstract}
This paper presents the design, implementation and measurements of a complete electronic front-end intended for high-resolution spatial detection of ion beams at counting rates higher than $10^{6}$ particles per second. The readout system is made up of three main multi-channel building blocks, namely: a transimpedance preamplifier, a signal-conditioning line receiver and a charge-to-digital converter, as well as some off-the-shelf components. The preamplifier and the line receiver have been specifically designed and optimized to minimize the overlapping probability of ion beams tracking, at high counting rates, in low-pressure gaseous secondary electron detectors. Experimental results are shown, considering $\alpha$ particles sources and particles beams, featuring an adaptive shaping time frame of 170-to-230 ns with a peak signal-to-noise ratio of up to $25 \mathrm{~dB}$. These performance metrics are competitive with the state of the art, demonstrating the suitability of the reported data acquisition and instrumentation system for precise and fast particle tracking detection.
\end{abstract}

\section{Index Terms}

Particle tracking, detectors, spatial detection, radioactive ions beams, readout electronics.

\footnotetext{
Manuscript received February 7, 2014, revised May 5, 2014, accepted July 9, 2014.
}

This work was partially supported by the Spanish Ministry of Economy and Competitiveness (with support from the European Regional Development Fund) under contracts CSD2007-00042, FPA2009 08848 and TEC2010-14825/MIC, and by "Consejería de Economía, Innovación y Ciencia y Empleo de la Junta de Andalucía” under contracts P07-FQM-02894, P12-TIC-1481.

A. Garzón-Camacho is with the Institute for the Structure of Matter (CSIC), C/ Serrano 113, 28006 Madrid, SPAIN, e-mail: alegarzon@us.es. B. Fernández is with the Centro Nacional de Aceleradores (CNA), Dpt. FAMN, Universidad de Sevilla, C/ Thomas Alba Edison, 7, Sevilla, SPAIN, e-mail: bfernand@us.es. M.A.G. Alvarez is with the Physics Institute of University of São Paulo, 66318 São Paulo, BRAZIL. J. Ceballos and J. M. de la Rosa are with the Institute of Microelectronics of Seville, IMSE-CNM (CSIC/Universidad de Sevilla), C/ Américo Vespuccio s/n, 41092 Sevilla, SPAIN, e-mail: jrosa@imse-cnm.csic.es. 


\section{A Fast Readout Electronic System for Accurate Spatial Detection in Ion Beam Tracking for the Next Generation of Particle Accelerators}

\section{INTRODUCTION}

Recent research studies in new isotopes like super-heavy nuclei and exotic nuclei (those with either an excess of neutrons or protons) has prompted the interest of the scientific community for the development of new instrumentation and measurement techniques in order to get a deeper understanding and knowledge of the nuclear structure and the reaction mechanisms behind these weakly bound radioactive elements with short half-lives, which can be produced at laboratory. To this purpose, the new generation of particle accelerators are able to produce low-energy Radioactive Ion Beams (RIB), with less than $10 \mathrm{MeV}$ per nucleon (MeV/u) [1], at counting rates higher than $10^{6}$ particles per second (pps). Due to the large angular aperture and low energy of these beams, their tracks must be detected before the target impact point, in order to accurately determine the incident energy of the particles which give rise to the nuclear reactions and to reconstruct the kinematics of such nuclear reactions fragments. To collect these data, a precise and fast electronic readout system is required for both spatial and time detection of the particles involved in the reactions, thus taking advantage of the huge amount of statistical information generated [2], [3].

Although a number of electronic readout systems have been developed in the past for nuclear detectors [4]-[17], very little has been done for the spatial detection in tracking of particles produced by low-energy RIB with $10^{6}$ counting rates. Indeed, the most common approach to implement the front-end electronic detection system is based on the conceptual architecture depicted in Fig. 1(a). In this scheme, a charge-sensitive preamplifier is used to amplify the input charge coming from the detector, and the preamplifier output is shaped and then digitized using a peak-sensing Analog-to-Digital Converter (ADC) [18].

The scheme in Fig. 1(a) has been mostly used for energy measurements [18], in which the relevant information - the amount of energy - is directly related to the value of the signal peak 


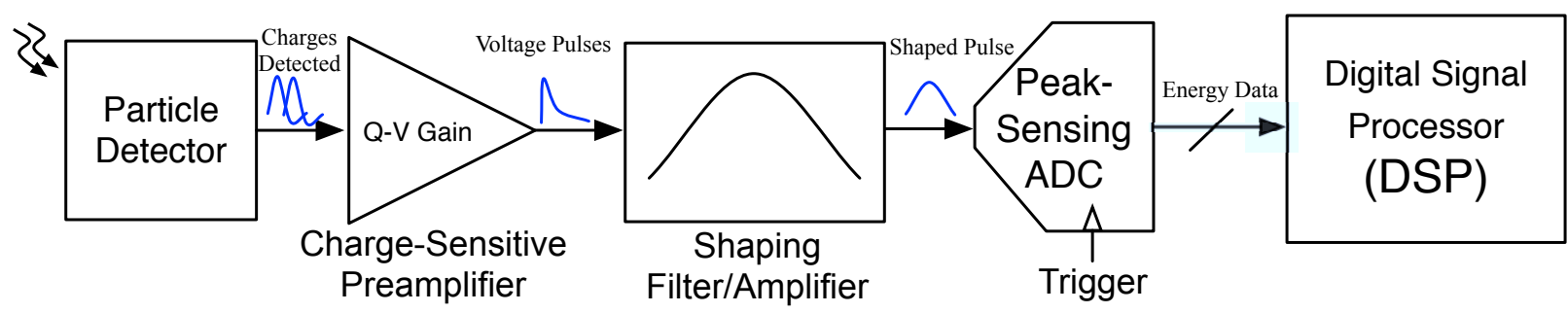

(a)

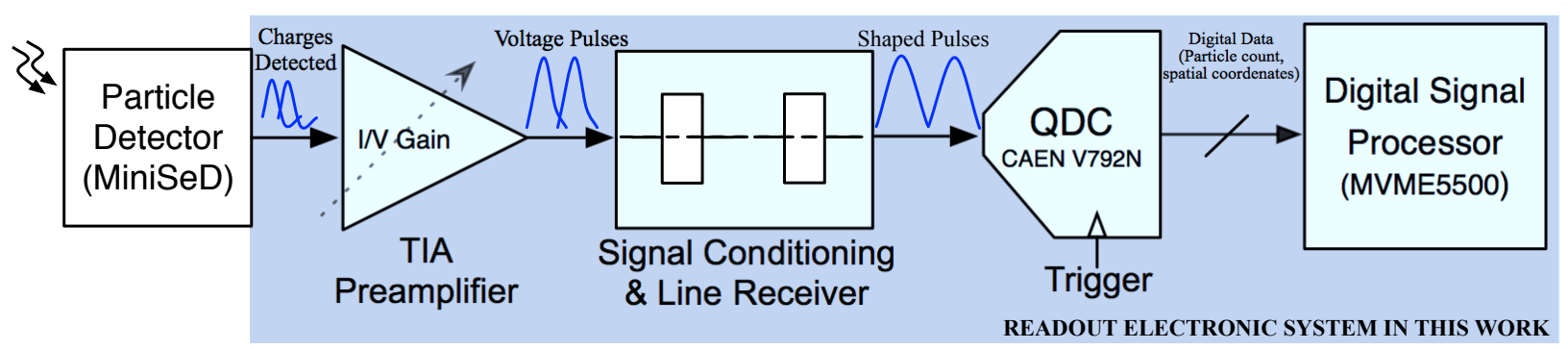

(b)

Fig. 1. Conceptual block diagram of an electronic readout system for particle detectors: (a) Based on a charge-sensitive preamplifier and a peak-sensing ADC. (b) Based on a preamplifier and a QDC (approach followed in this work).

amplitude, instead of the duration of an event. Indeed, this piece of information - not only related with the value of the charge detected but with the time interval in which is detected - is destroyed by the charge sensitive amplifier, thus making the detection of two consecutive particles more difficult as either the counting rate or the input charge from the detector are increased [4].

An alternative implementation of the readout system is conceptually depicted in Fig. 1(b), where a fast TransImpedance preAmplifier (TIA) is used at the front-end interface connected to the detector, and its output signal, after some signal conditioning, is digitized by a Chargeto-Digital Converter (QDC) [18]. One of the most challenging circuits in the readout system of Fig. 1(b) is the preamplifier, due to its early position in the chain. This has motivated the development of different alternative implementations of this building block [4], [8], [10], [19]-[21]. To the best of the authors' knowledge, reported solutions were only focused on the partial implementation of some blocks in Fig. 1(b). However, these solutions are not suitable to discriminate between two consecutive events when the occurrence rates are higher than $10^{6} \mathrm{pps}$, and correspond to particles produced by low-energy $(<10 \mathrm{MeV} / \mathrm{u})$ ion beams. 
This paper contributes to this topic and proposes a complete readout electronic system capable of detecting particles at counting rates over $10^{6}$ pps with a peak Signal-to-Noise Ratio (SNR) larger than $25 \mathrm{~dB}$. The system is intended to cope with the specifications of spatial measurement in low-pressure gaseous Mini-Secondary electrons Detectors (Mini-SeD) [2], [3], [22], although the presented techniques, design methodologies and circuit strategies can be applied to other detectors with similar physical conditions and electrical specifications. The presented system is based on the scheme of Fig. 1(b), and combines off-the-shelf electronic components - like the QDC - with other building blocks (preamplifier and line receiver) which have been specifically synthesized and designed to cope with target specifications. A systematic top-down/bottom-up design/verification methodology has been followed to optimize the performance of the overall system for target specifications. A number of measurements - considering different experiment conditions and production sources - are shown to validate the presented approach.

The paper is structured as follows. Section II describes the architecture of the proposed readout electronic system, giving an overview description of Mini-SeDs and the electrical system-level specifications. Section III presents the design and implementation of main building blocks, namely the preamplifier and the line receiver. Finally, experimental results are given in Section IV and conclusions are drawn in Section V.

\section{ReAdout ARChitecture AND System-LEVEl SpecificAtions}

As stated in the Introduction, the proposed readout electronic system is based on the conceptual scheme depicted in Fig. 1(b), intended to detect particles at a rate over $10^{6} \mathrm{pps}$ in a Mini-SeD. The physical characteristics of this detector will determine the electrical specifications and the specific topology required for the different building blocks embedded in the readout electronics.

\section{A. Background on Mini-SeDs}

Mini-SeD is a kind of gas ionization multi-wire chamber that works at low pressure and has demonstrated to be very suited for the detection of low-energy ion beams with large angular and energy straggling, thus allowing an accurate reconstruction of the trajectory (tracking) - position and Time of Flight $(\mathrm{ToF})$ - of individual particles at high production rates before the nuclear reaction. 


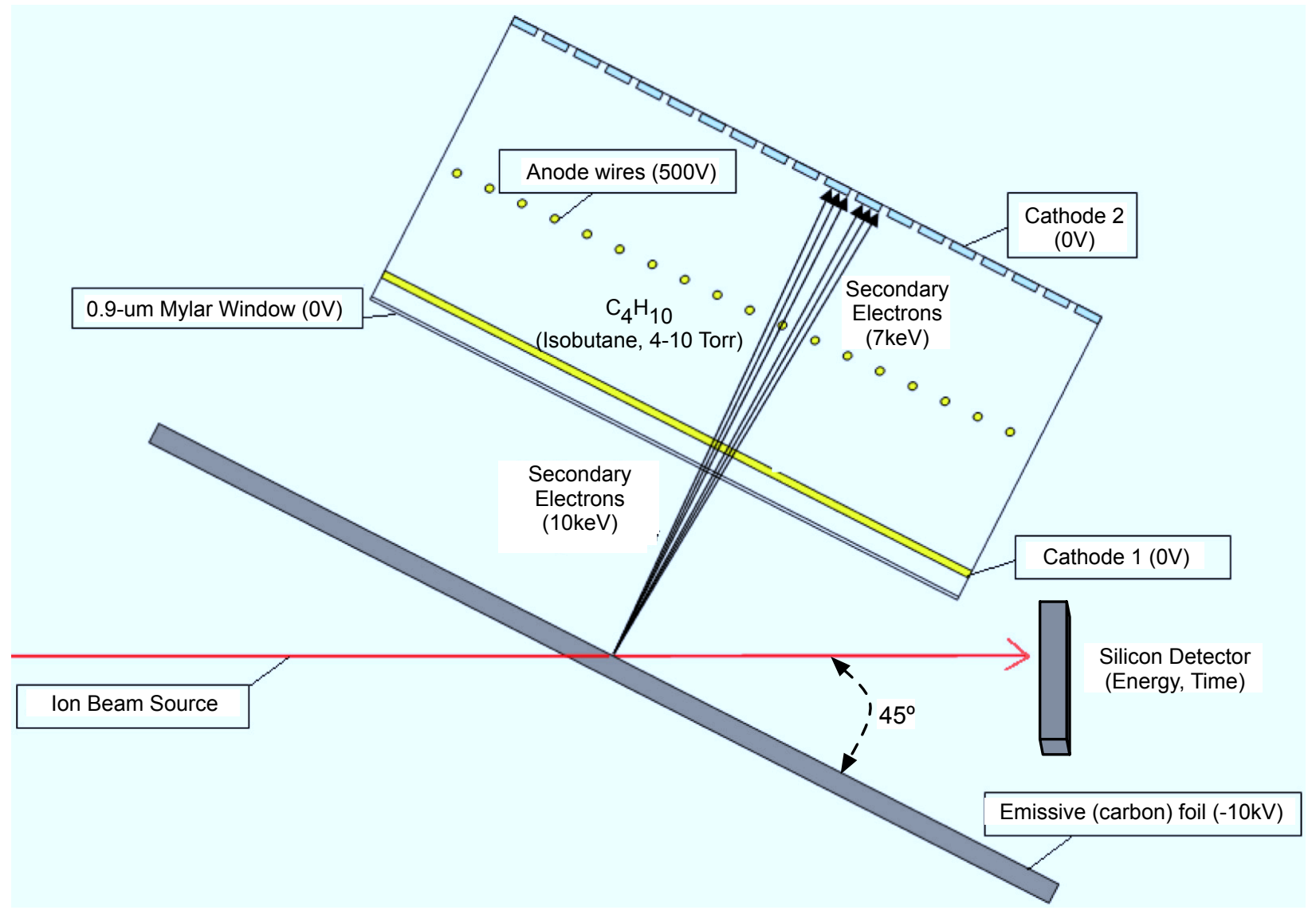

Fig. 2. Illustrating the operating principle of the Mini-SeD.

The structure and working principle of a SeD is detailed in [2], [22] and conceptually illustrated in Fig. 2. Essentially, the complete detection device is made up of three main parts, namely: an emissive foil, an electrostatic guiding and focalizing system and a detector prototype Mini-SeD [3]. The latter is composed of an anode placed between two grounded cathodes. The anode consists of a wire plane, where each wire has a diameter of $20 \mu \mathrm{m}$. One of the cathodes is a wire plane (72 wires linked in an 3-by-3 array) of $50 \mu \mathrm{m}$ diameter, and the other is made of 28 copper strips implemented in a Printed Circuit Board (PCB) with 2.54 pitch. This way, the low-energy heavy ions pass through the emissive foil (placed at $45^{\circ}$ respecting to the beam line at $0^{\circ}$ ) and the secondary electrons generated in the collisions are accelerated by an electrical field and focused by a longitudinal magnetic field towards the detector, which can be filled with different gas mixtures at low pressure (typically from 4 to 10 Torr) for developing proposals.

The position in particle tracking is characterised by the spatial coordinates, which are in turn 
obtained by measuring and analysing the center of gravity of the data generated by the charge distribution induced in the cathodes by the movement of the charge created inside the detector in an avalanche process. The ToF of the ions can be calculated from the anode signals of two different Mini-SeDs in the same beam line. The resulted time signals from the anode - after some amplification and conditioning - can be also used to trigger the QDC in Fig. 1, as will be shown later. The precise characterisation of particles tracking - in terms of the spatial coordinates - strongly depends on the nature of charge signals provided by the detector cathodes, which indeed constitute the input signals for the readout electronic system driving the detector.

\section{B. Readout Electronic Specifications}

The Mini-SeD can be modeled as a current source for the behavioral high-level simulation of the readout electronic system to be designed. The most important electrical parameters that characterize the current signal provided by the detector cathodes are the following: the peak current signals at each of the 64 cathodes, $I_{C}$; the rise/fall time, $t_{r, f}$, and the average time of an event, i.e. the arrival time of a particle, which is the inverse of the particles production rate $-1 \mu \mathrm{s}$ in the case of $10^{6} \mathrm{pps}$ counting rate.

In order to obtain the most precise values for $I_{C}$ and $t_{r, f}$, the Mini-SeD was simulated using GEANT4 - a CAD tool for the modelling and simulation of particle detectors [23], [24]. To this purpose, the dimensions and physical properties of the different materials used in the detector were included in the simulator, together with some of the physical conditions in which the Mini-SeD is going to operate - summarized in Table I.

Unfortunately, the model used in GEANT4 does not take into account some important limiting

TABLE I

Physical Parameters of The Mini-SeD in GEANT4

\begin{tabular}{|l|c|}
\hline Parameter & Value \\
\hline Energy of the incident beam & $1 \mathrm{MeV} / \mathrm{u}$ \\
\hline Mylar window thickness & $0.9 \mu \mathrm{m}$ \\
\hline Emissive foil voltage & $-10 \mathrm{kV}$ \\
\hline Magnetic field & $100 \mathrm{G}$ \\
\hline Electric field & $600 \mathrm{~V} / \mathrm{m}$ \\
\hline
\end{tabular}


factors like the avalanche currents produced at the active areas of the detector, the current induced by the secondary electrons at the cathodes, and the parasitic impedances and capacitances at the cathodes. These effects must be taken into account in order to obtain an accurate estimation of the electrical parameters of the current source. To this end, the behavioral model parameters used to model the Mini-SeD were completed and refined by comparing system-level simulations with measurements taken on the Mini-SeD when tested together with an older electronic readout equipment at CEA-Saclay laboratories [19], [25]. Multiple system-level simulations were carried out in order to match the results obtained by the experiments under different conditions and production sources. According to these experimental tests and the simulations carried out using GEANT4, it was concluded that the Mini-SeD can be modeled as a current pulse source with the following parameters: pulse amplitude, $I_{C}=23 \mu \mathrm{A}$, and rise/fall time, $t_{r, f}=10 \mathrm{~ns}$. These parameters constitute the system-level specifications for the electronic readout system developed in this work and described in the next section.

\section{Block diagram of the proposed readout electronic system}

Fig. 3 shows the architecture of the proposed readout electronic detection system, which is intended to cope with the system-level specifications of the input current signal provided by the Mini-SeD cathodes. To this end, the Mini-SeD - placed inside a vacuum chamber - is connected to eight 4-channel ${ }^{1}$ transimpedance preamplifiers, which are driven by four 8-channel signalconditioning line receivers that accommodate the signal ranges to be discriminated and digitized by two 16-channel V792N QDC modules from CAEN. The resulting digital data is finally processed by a MVME5500 single-board processor from Motorola, and statistically analyzed by using Multi Instance Data Acquisition System (MIDAS) [26]. This way, the presented system is able to extract the information related to the spatial coordinates $(X, Y)$ in the trajectory followed by the particles, detected in a 16x16 main grid formed by the two cathodes of the Mini-SeD (see Fig. 2).

\footnotetext{
${ }^{1}$ The number of channels was chosen to adapt the presented readout electronics to the test equipment and experimental conditions available at CEA-Saclay laboratories, where the first measurements were carried out and compared with previous preamplifiers and electronic readout systems. However, such a number of channels can be adapted to the required measurement set-up, based on the same architecture shown in Fig. 3.
} 


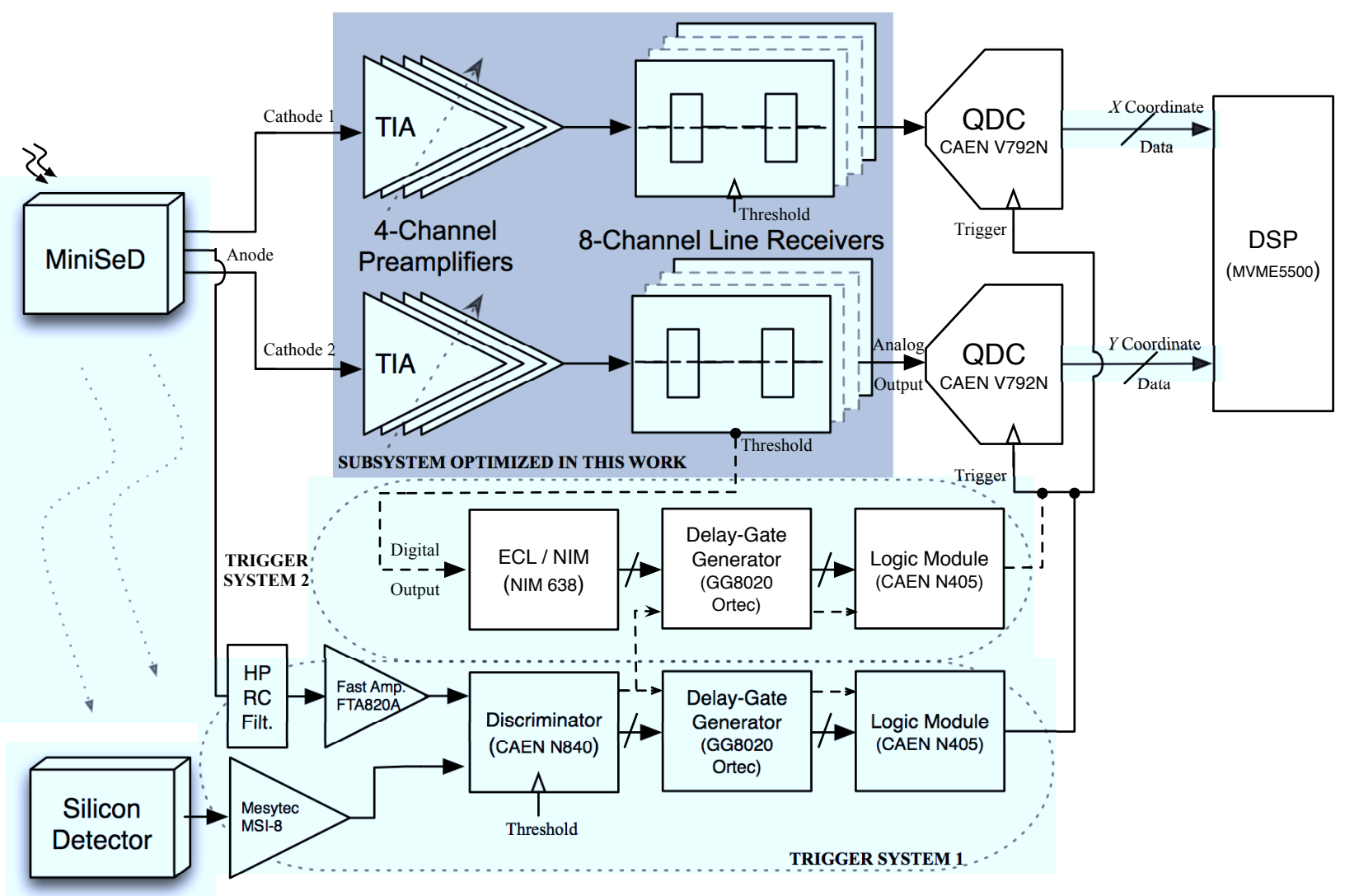

(a)

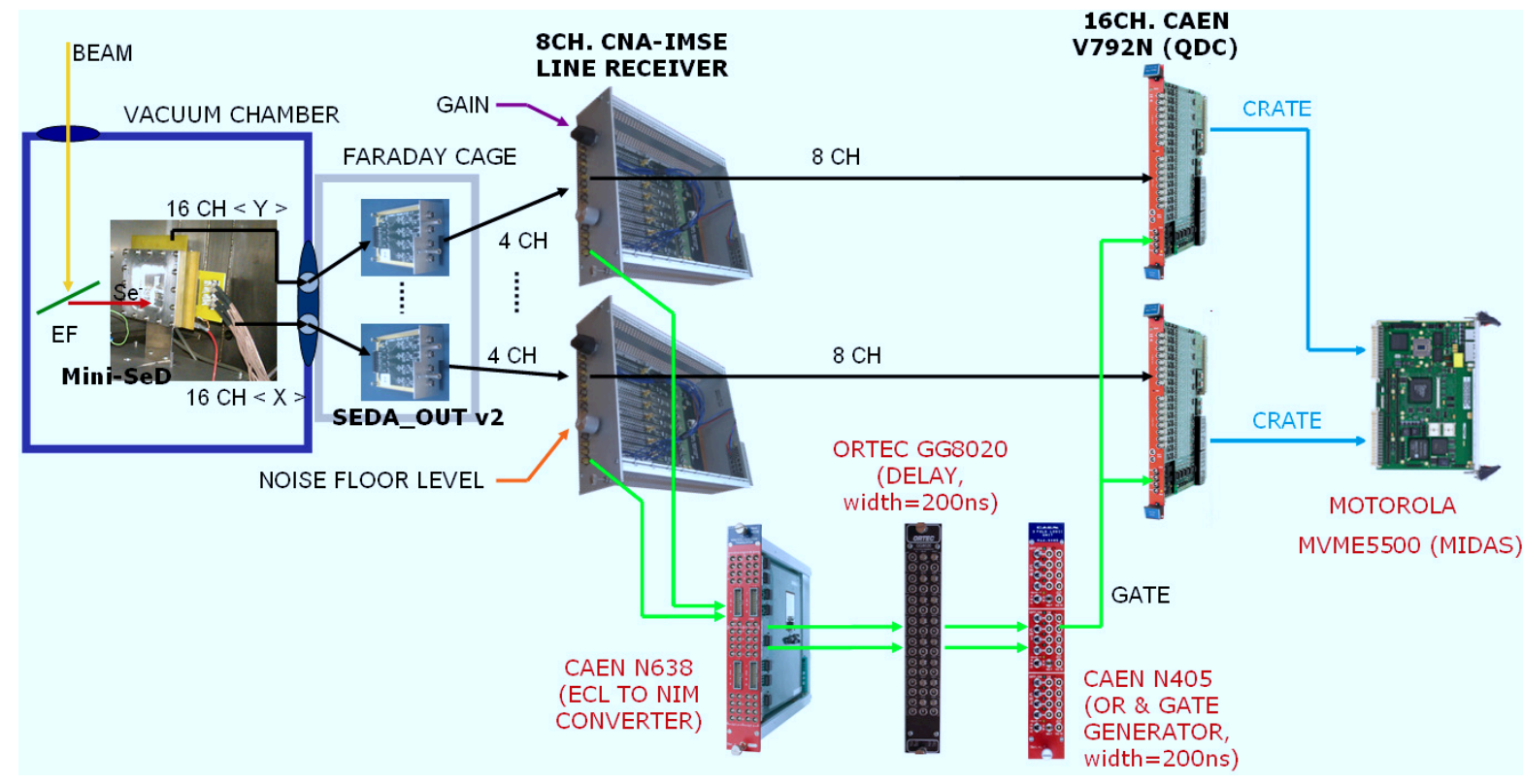

(b)

Fig. 3. Proposed readout system. (a) Conceptual architecture. (b) Block diagram highlighting the main parts and subcircuits. 
In order to detect valid events, the QDC is triggered (depending on the detection system setup) only when a real particle is detected. To this purpose, the trigger signal can be generated by capturing the information related to the particles detected by both the anode of MiniSeD and a silicon detector. Then, a specific 200-ns pulse-width signal waveform is generated by the trigger system as the time window of the QDC for integrating the detected charge signal. This trigger system - denoted as Trigger System 1 in Fig. 3(a) - is made up of several parts. On the one hand the signal provided by the silicon detector is amplified by a 8-channel preamplifier (MSI8 from Mesytec). On the other hand, the signal provided by the Mini-SeD anode is filtered by a second-order RC filter, which acts as a low-pass filter for the supply voltages and as a high-pass filter for the anode output (see Fig. 3(a)). Both detected signals are compared with a threshold voltage in a 8-channel discriminator (N840 from Caen), which is used as the input for a delay-gate generator GG8020 from Ortec and, finally to a logic module N405 from Caen, that accommodates the data to trigger the QDC only when particles are detected by both detectors at the same time, thus discriminating valid events from noise with an appropriate SNR. An alternative trigger generator - denoted as Trigger System 2 in Fig. 3(a) - can be used. This system is similar to the Trigger System 1, although the additional silicon detector is not used, since it is based on a digital control signal provided by the line-receiver.

All the off-the-shelf components in the presented readout system of Fig. 3(b) - whose main electrical characteristics ${ }^{2}$ are summarized in Table II - have been properly selected to cope with the required specifications of the particles spatial detection with Mini-SeD. However, due to the very demanding specifications for the preamplifier and the line receiver - in terms of SNR and transient response - to detect low-amplitude $(<23 \mu \mathrm{A})$ current-mode signals with $t_{r, f}=10 \mathrm{~ns}$, an specific design is carried out to optimise the performance of these subsystems according to these input signal and spatial detection system characteristics.

\section{Design of Main Building Blocks}

The 4-channel preamplifier used in the readout system - referred to as Secondary Electrons Detector preAmplifier (SEDA) in Fig. 3(b) - has been designed to process charge signals coming from the Mini-SeD cathodes, tracking high counting rates of RIB with around $1 \mu$ s of average

\footnotetext{
${ }^{2}$ Datasheets of all off-the-shelf IC parts used in the proposed readout system are available online.
} 
TABLE II

ELECTRICAL CHARACTERISTICS OF THE MAIN READOUT BUILDING BLOCKS

\begin{tabular}{|l|l|c|}
\hline Building Block & Parameter & Value \\
\hline QDC (V792N) & Input impedance & $50 \Omega$ \\
& Analog input range & {$[-1 \mathrm{~V}, 15 \mathrm{mV}]$} \\
& Fixed Transient Delay & $15 \mathrm{~ns}$ \\
\hline \multirow{2}{*}{ ELC-NIM Conv. (N638) } & ECL logic zero range & {$[-1.95,-1.48] \mathrm{V}$} \\
& ECL logic one range & {$[-1.17,-0.84] \mathrm{V}$} \\
& NIM logic zero range & {$[-0.20,1.00] \mathrm{V}$} \\
& NIM logic one range & {$[-1.80,-0.60] \mathrm{V}$} \\
\hline \multirow{2}{*}{ Delay-Gate Gen. (CG8020) } & Transient delay & $2 \mathrm{~ns}$ \\
\hline Lutput delay range & {$[70 \mathrm{~ns}, 1 \mu \mathrm{s}]$} \\
\hline \multirow{2}{*}{ Logic Module (N405) } & Fixed Transient delay & $20 \mathrm{~ns}$ \\
\hline & Pulse width range & {$[20,800 \mathrm{~ns}]$} \\
& Fixed Transient delay & $14 \mathrm{~ns}$ \\
\hline
\end{tabular}

arrival time between two consecutive particles. In order to relax the bandwidth specifications, the current signals waveforms coming from the cathodes - with peak amplitudes of $23 \mu \mathrm{A}$ and $t_{r, f}=10 \mathrm{~ns}-$ are shaped so that they can be digitized by the QDC during an integration time period of $200 \mathrm{~ns}$, i.e. a $20 \%$ of the average particles arrival time. This way, the number of events can be modelled as a Poisson distribution whose main parameters are $\lambda=1 \mu$ s and $K=200 \mathrm{~ns}$, with $\lambda$ and $K$ being the average arrival time and the integration time, respectively. Thus, there will be overlapping if two consecutive particles are detected within a 200ns time frame. Taking this into account and that the detector output signals are current pulses with 20ns duration, this constitutes less than $2 \%$ of overlapping probability between two consecutive valid events. Another important design specification is the SNR, which should be high enough to discriminate current peaks generated in the cathodes when particles are detected, typically in the order of a few $\mu \mathrm{A}$. Finally, other two important design parameters to be considered are the output impedance of $50 \Omega$ and the input parasitic capacitance of $30 \mathrm{pF}$. The latter is limited by the cables used to 
connect each preamplifier channel - outside the vacuum chamber (see Fig. 3) - to the detector ${ }^{3}$.

\section{A. Preamplifier Topology and Simulated Performance}

Fig. 4 shows the schematic of one of the four (identical) channels of the proposed preamplifier. The circuit is composed of a TIA subcircuit, a shaper filter, an ac-coupled inverting amplifier and a line driver. An ElectroStatic Discharge (ESD) diode-based protection circuit is placed at the input node to avoid damages caused by high voltage sparks and other experimental set-up interference signals. The different preamplifier Integrated Circuit (IC) parts have been chosen to fulfill the required electrical specifications detailed above. To this purpose, a simulation-based design process has been followed from system-level specifications to building-block specifications and final physical (PCB) implementation. This way, a preliminary selection of several ICs was considered according to the electrical characteristics detailed in their data sheets, and they were simulated in order to select the most appropriate components that satisfy the overall system performance in terms of the required SNR and speed specifications.

The TIA in Fig. 4 was implemented using the AD8015 IC from Analog Devices. This circuit transforms the current signals coming from detector cathodes into voltage signals with a transimpedance gain of $80 \mathrm{~dB} \Omega$ within a $240-\mathrm{MHz}$ bandwidth, keeping the same value of the input $t_{r, f}$ and minimizing the probability of overlapping signals. In this configuration, this building block has an input referred noise spectral density of $3 \mathrm{pA} / \sqrt{\mathrm{Hz}}$. The effects of parasitic capacitance on both signal bandwidth and noise figure have been taken into account in the simulations. Thus, considering a $30-\mathrm{pF}$ input parasitic capacitance due to the cable, the TIA output total noise power is $3.35 \mathrm{mVrms}$ within a $32.7-\mathrm{MHz}$ signal bandwidth - in good agreement with the overall electronic front-end specifications described in Section II.

Another important block of the preamplifier system in Fig. 4 is the shaper filter, which is needed to slow down the preamplifier output signal. This block is implemented by a biquad filter configuration made up of a RC High-Pass (HP) filter, a buffer and a second-order Low-

\footnotetext{
${ }^{3}$ A PRO POWER RG179 coaxial cable with $40-\mathrm{cm}$ length and $64 \mathrm{pF} / \mathrm{m}$ parasitic capacitance was used to connect the Mini-SeD - placed inside a vacuum chamber - and the proposed readout system. The overall load capacitance of 30pF, was estimated from the coaxial-cable capacitance and those parasitic capacitances associated to the corresponding connectors - approximately $2 \mathrm{pF}$ each one. Simulations carried out by using this lumped capacitor model - instead of considering the cable as a transmission line - allow us to accurately predict the parasitics effects associated to the cable.
} 


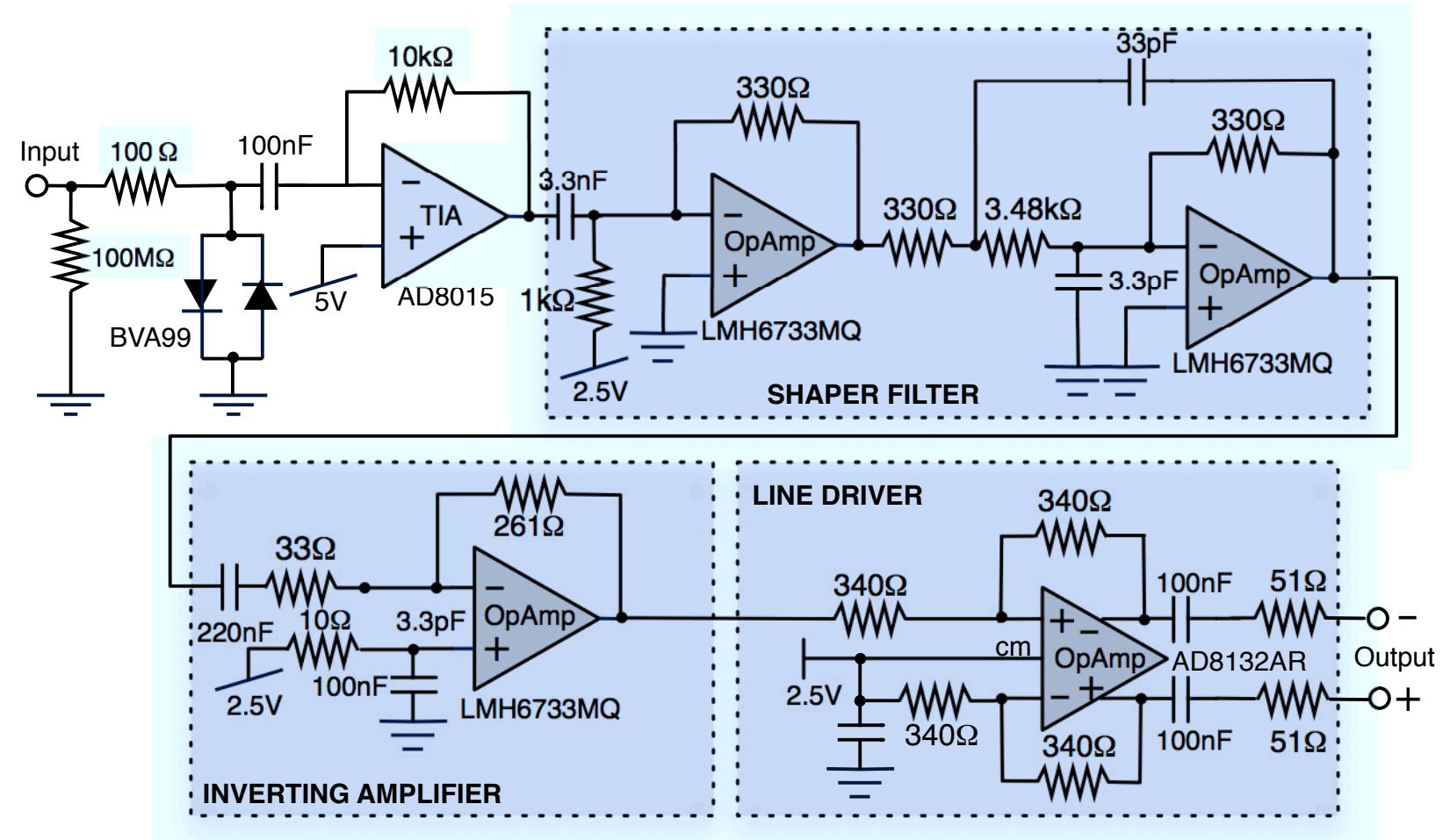

Fig. 4. Schematic of the one of the channels of the proposed preamplifier system (referred to as SEDA).

Pass (LP) filter. The cut-off frequency of the RC HP filter was set to 50-kHz in order to reduce the TIA output offset error and flicker noise. Both the buffer and the biquad filter were implemented using LMH6733 operational amplifiers configured with 0-dB gain, yielding a shaping time lower than 200ns - in agreement with the required speed performance.

The gain of the preamplifier is provided by the fourth block in Fig. 4, implemented as an ac-coupled inverting amplifier based on the LMH6733MQ operational amplifier. This inverting configuration was designed and tested to have a variable gain from $0 \mathrm{~dB}$ to $18 \mathrm{~dB}$, by properly changing the feedforward and feedback resistors connected to the opamp.

The last building block used in the preamplifier chain is a differential line driver. This block - implemented by using an AD8132 opamp - was designed to properly transmit the required high-speed differential signals over $50 \Omega$ coaxial cables connecting the preamplifier and the line receiver in Fig. 3, thus minimizing the line reflections as well as the common-mode and ground noise.

In order to validate the preamplifier performance, a number of simulations of the circuit in 
TABLE III

SIMULATED PERFORMANCE OF THE SHAPER FILTER FOR DIFFERENT VALUES OF THE LPF CUT-OFF FREQUENCY

\begin{tabular}{|l|c|c|c|c|c|}
\hline Cut-off Freq. (MHz) & $\mathbf{1 0}$ & $\mathbf{1 5}$ & $\mathbf{2 0}$ & $\mathbf{2 5}$ & $\mathbf{3 0}$ \\
\hline \hline Total Noise (mVrms) & 1.60 & 1.61 & 1.63 & 1.68 & 1.78 \\
\hline Peak Voltage (mV) & 54.0 & 81.0 & 102.0 & 118.0 & 130.0 \\
\hline Peak SNR (dB) & 30.6 & 34.1 & 35.9 & 36.9 & 37.3 \\
\hline Fall/Rise time (ns) & $38 / 92$ & $35 / 63$ & $33 / 37$ & $30 / 27$ & $29 / 26$ \\
\hline Shaping time (ns) & 300 & 200 & 170 & 160 & 160 \\
\hline
\end{tabular}

TABLE IV

SIMULATED PERFORMANCE OF THE PREAMPLIFIER FOR DIFFERENT INVERTING AMPLIFIER GAINS AND 20-MHZ LPF CUT-OFF FREQUENCY

\begin{tabular}{|l|c|c|}
\hline Inverting amp. gain & $\mathbf{4}$ & $\mathbf{8}$ \\
\hline \hline Total Noise (mVrms) & 3.28 & 6.57 \\
\hline Peak SNR (dB) & 35.7 & 35.7 \\
\hline Peak Voltage (mV) & \pm 200 & \pm 400 \\
\hline Fall/Rise Time (ns) & $33 / 37$ & $33 / 37$ \\
\hline Shaping Time (ns) & 170 & 170 \\
\hline
\end{tabular}

Fig. 4 were carried out using NI Multisim ${ }^{\mathrm{TM}}$ [27], considering the SPICE models of all circuit components and building blocks, thus allowing us to make accurate simulations of the overall system. To this end, a 30-pF input parasitic capacitance and a current source with the same characteristics as Mini-SeD output current waveform signals, i.e., $t_{r, f}=10 \mathrm{~ns}, 23-\mu \mathrm{A}$ current peak and $1-\mu$ s period, were considered. Table III sums up the simulated performance, showing the main features for different values of the LP cut-off frequency at the shaper output. The $20-\mathrm{MHz}$ bandwidth configuration was chosen to optimize the trade-off between the required SNR and shaping time. Using this configuration, the main simulated performance metrics of the preamplifier are summarized in Table IV. As an illustration, Fig. 5 shows the transient response of one channel of the preamplifier considering an input current pulse, by depicting the output signal waveforms at the output of the main building blocks, and showing a correct performance of the circuitry. 


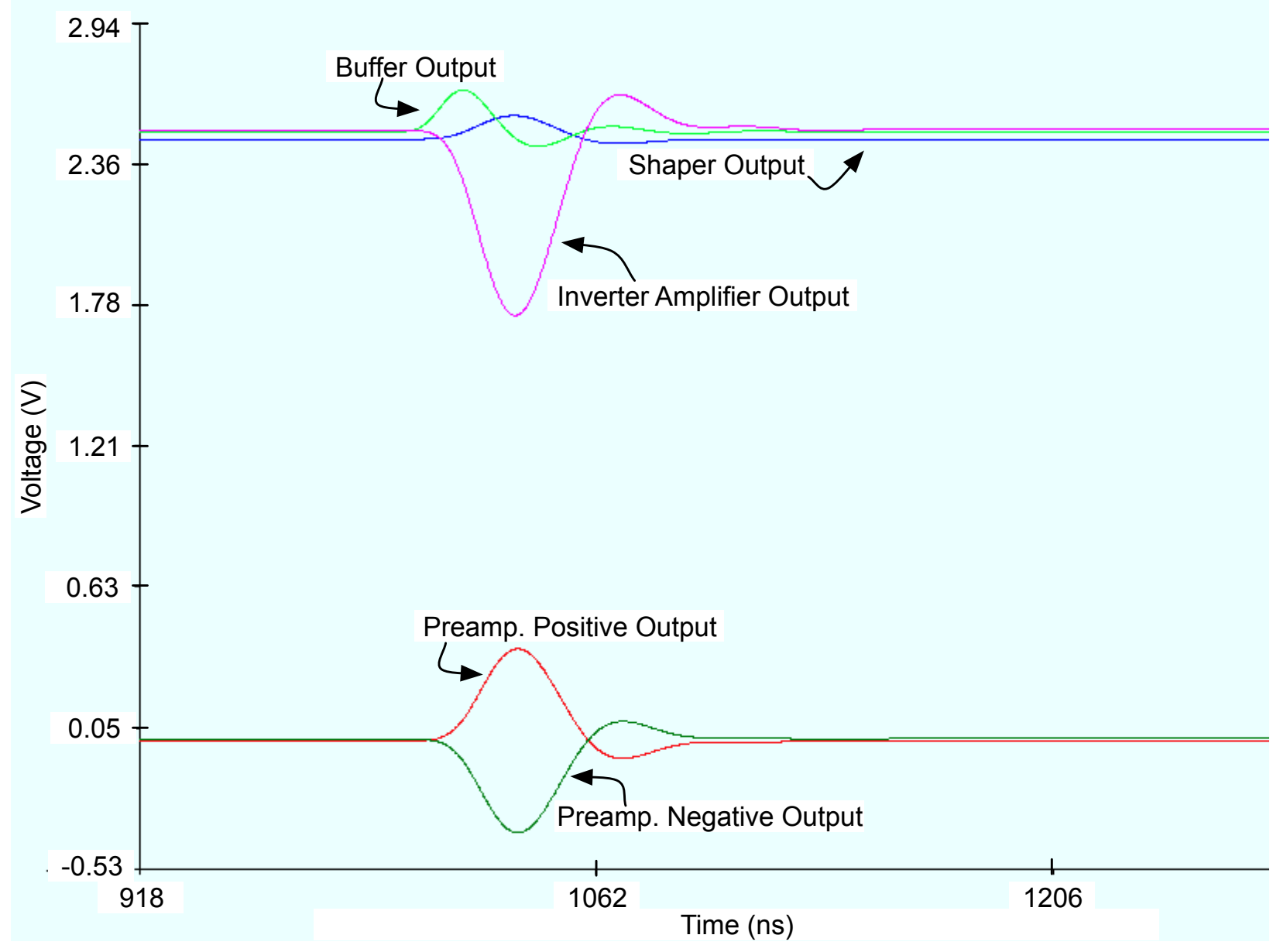

Fig. 5. Illustrating the simulated performance of the preamplifier. Note that the TIA output waveform is the same as the buffer output - depicted in this figure - except for the DC level (3.7V for the TIA output and $2.5 \mathrm{~V}$ in the case of buffer output.)

\section{B. Line Receiver}

As stated in previous sections, a line receiver is used to adapt the output signals provided by the preamplifier to the electrical requirements of the QDC in Fig. 3.

Fig. 6 shows the conceptual schematic of one of the channels of the line receiver. It is made up of three main building blocks, namely: a programmable-gain differential amplifier, a comparator and a LC passive filter. The front-end differential amplifier, implemented with an AD830JRZ IC part, transforms the fully-differential input signal into a single-ended signal, and adapts the signal range to the QDC with a programmable voltage gain. This gain can be reconfigured from 1 to 8 by properly selecting the feedback resistor to be $1 \Omega, 100 \Omega, 300 \Omega$ and $700 \Omega$, with respect 


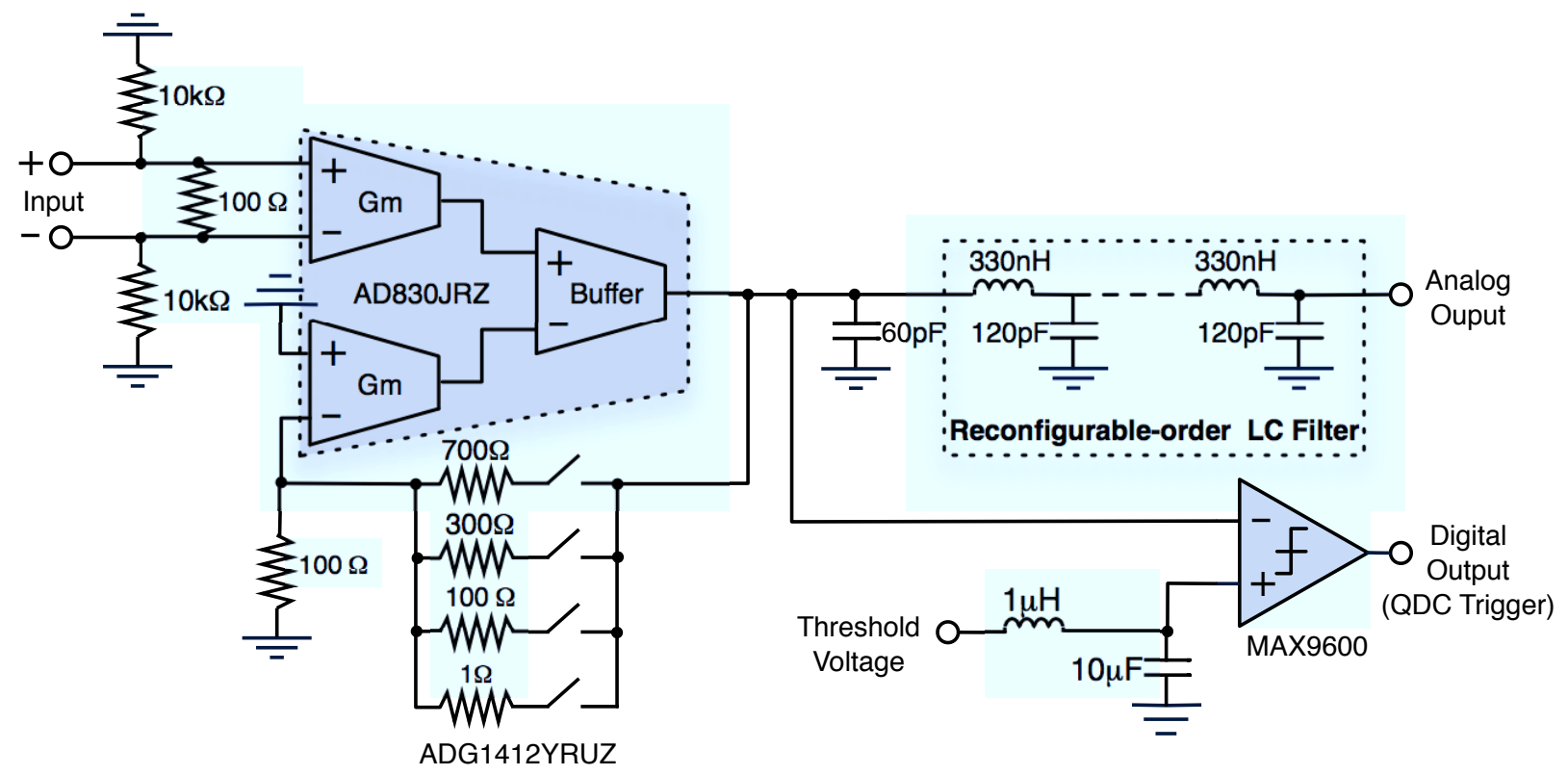

Fig. 6. Schematic of the one channel of the proposed line receiver.

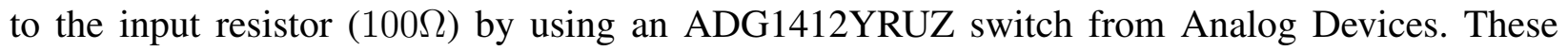
component values were chosen in order to get the desired performance even considering the impact of circuit parasitics.

The output of the line-receiver differential amplifier is used as an input to the comparator in order to generate a trigger signal that activates the QDC (see Fig. 3(a)) only when a valid event (particle) is detected. This way, the comparator output provides a logic one if the amplifier output voltage is high enough compared to a tunable threshold voltage in order to guarantee that a valid event has been detected. Otherwise, the comparator output is a logic zero. The logic levels are set according to the Emitter-Coupled-Logic (ECL) input range and noise margins of the ECL-NIM ${ }^{4}$ converter, thus being within $[-0.84,-1.17] \mathrm{V}$ for a logic one and $[-1.48,-1.95] \mathrm{V}$ for a $\operatorname{logic}$ zero, which are converted to the NIM logic digital input (Gate) of the QDC.

The last block in Fig. 6 is a LC passive filter, which is used to synchronise both the digital input signal of the QDC (Gate) with the analog output of the differential amplifier, so that the latter signal can be properly processed by the QDC within the time interval in which it is activated when a valid event is detected $-200 \mathrm{~ns}$ in our case. To this purpose, the filter order can

\footnotetext{
${ }^{4}$ The term NIM logic is normally used to denote Nuclear Instrumentation Module logic.
} 


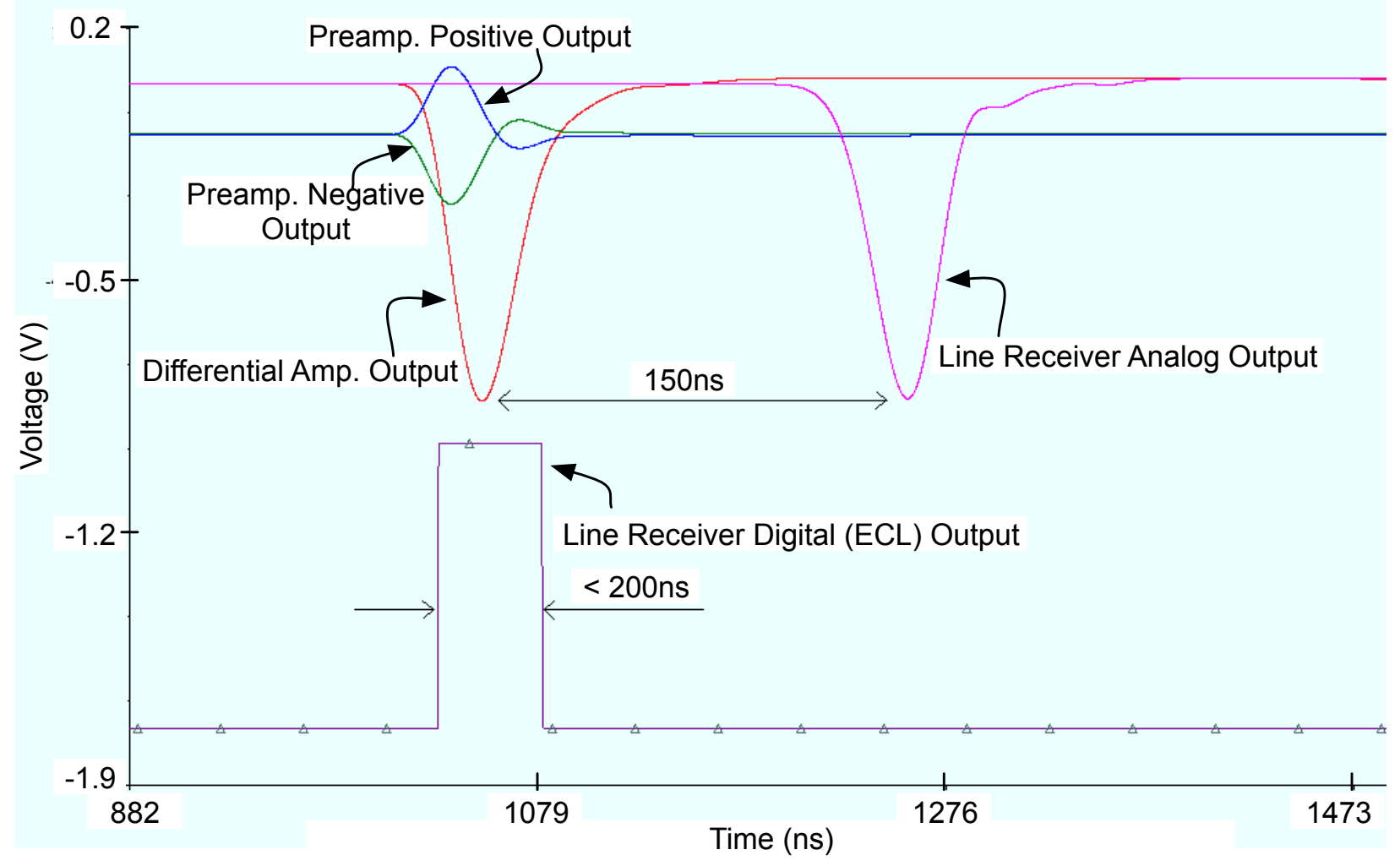

Fig. 7. Illustrating the simulated performance of the line receiver with unity gain.

be configured to achieve the required delay between both signals. For instance, a 150-ns delay can be obtained in our case with an LC filter of 23-rd order. This way, the gate signal delay can be tuned by the GG8020 block in order to synchronise that signal with the analog output of the line receiver (analog input of the QDC). Note that, as the input impedance of the QDC is $50 \Omega$, the LC filter must be designed to match this impedance within the required frequency range, i.e. from $50 \mathrm{kHz}$ to $20 \mathrm{MHz}$. As an illustration, Fig. 7 shows the simulated waveforms at the output of main blocks of the line receiver, when it is excited by a preamplifier output signal of $\pm 400 \mathrm{mV}$ peak voltage, demonstrating a correct timing and synchronization. Table $\mathrm{V}$ sums up the simulated performance of the line receiver, showing its main features for different differential amplifier gains.

\section{System IMPLEMENTATION AND MEASUREMENTS}

The proposed readout system has been designed to work outside the vacuum chamber, in which the Mini-SeD is placed. The connection between the detector and the electronic front- 
end is shown in Fig. 8(a), illustrating the detailed implementation of the 4-channel preamplifier (Fig. 8(b)) and the 8-channel line receiver (Fig. 8(c)). A number of experiments have been carried out in order to verify the performance of the presented readout system, considering the signal stimuli and environment conditions detailed in the next sections.

\section{A. Pulse Generator Experiment}

The first experiment was based on a pulse generator input signal, which emulates the input signal applied at the anode of the Mini-SeD. To this purpose, the instrumentation set-up shown in Fig. 9 was considered. An impulse signal waveform, with a peak amplitude of 0.45-V, 20-ns rise-time and 10-ms period, was used to induce the same quantity of charge in the Mini-SeD cathodes, thus emulating a particle detection event.

The cathodes output current signals were transmitted by the cables to the feedthrough connectors installed on the flange of the vacuum chamber, which has been developed for connecting detectors inside with the readout system outside the chamber. Thus, after being preamplified, fully-differential signals with \pm 200 -mV peak voltage and 150-ns shaping time are obtained.

Fig. 10 illustrates the analog output waveforms obtained for different values of the differentialto-single-ended gain programmed from 1 to 8 . It can be shown how the output signal of the proposed line receiver has a reconfigurable gain, $t_{r, f}$ and shaping time of 1.2-to-2.4, 70-to228ns and 180-to-330ns, respectively. These waveform characteristics are in good agreement with predicted simulation performance and are very appropriate to be digitized by the QDC.

Note that the QDC needs to be activated only when a real event is detected. This is illustrated in Fig. 11, that shows the digital gate signal generated by the trigger system 2 (see Fig. 3(b)) with the digital output signal of the line receiver. Note that the analog signal can be perfectly

TABLE V

SIMULATED PERFORMANCE OF THE LINE RECEIVER FOR DIFFERENT VALUES OF THE DIFFERENTIAL AMPLIFIER GAIN

\begin{tabular}{|l|c|c|c|c|}
\hline Differential amp. gain & $\mathbf{1}$ & $\mathbf{2}$ & $\mathbf{4}$ & $\mathbf{8}$ \\
\hline Peak Voltage (V) & -0.7 & -1.16 & -1.67 & -2.11 \\
\hline Overall Gain & 0.88 & 1.45 & 2.10 & 2.63 \\
\hline Shaping Time (ns) & 150 & 160 & 185 & 290 \\
\hline SNR (dB) & 34.7 & 33.9 & 30.6 & 26.1 \\
\hline
\end{tabular}




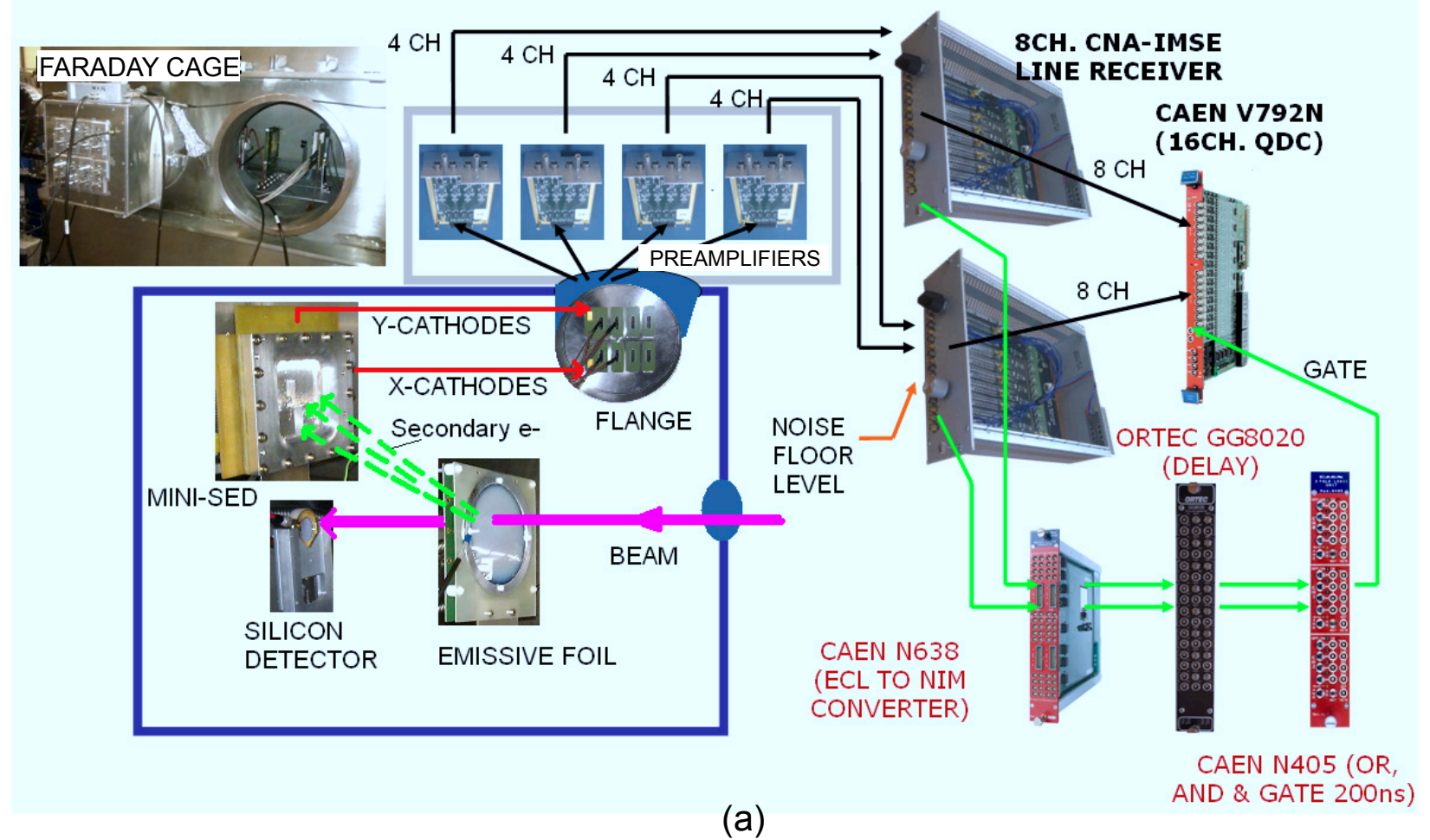

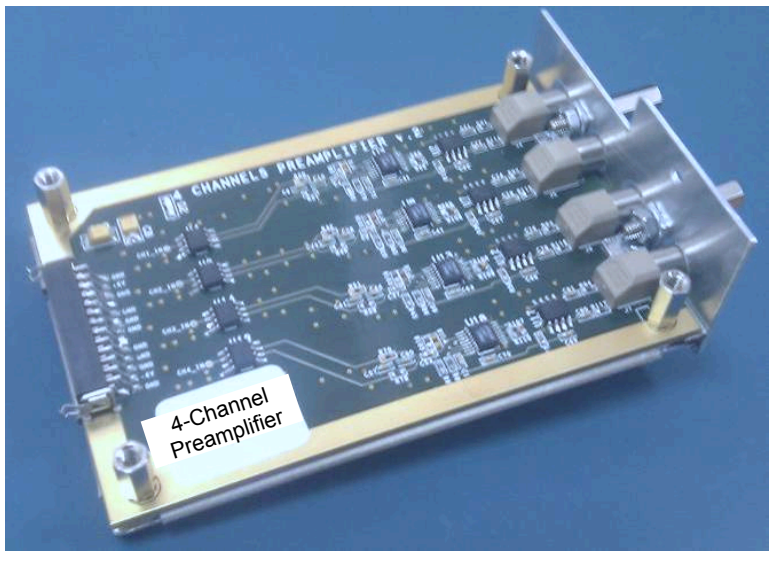

(b)

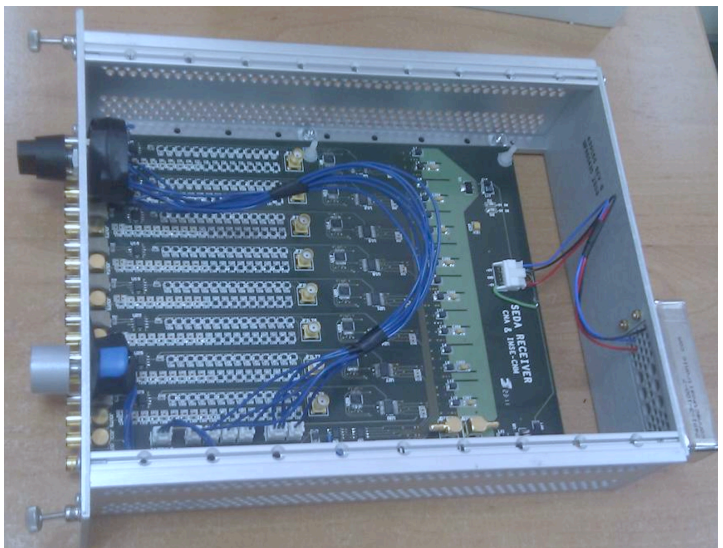

(c)

Fig. 8. Implementation of the proposed readout system and measurement set-up. (a) Connection with the vacuum chamber including the Mini-SeD. (b) Preamplifier PCB subsystem. (c) Line receiver PCB subsystem. 


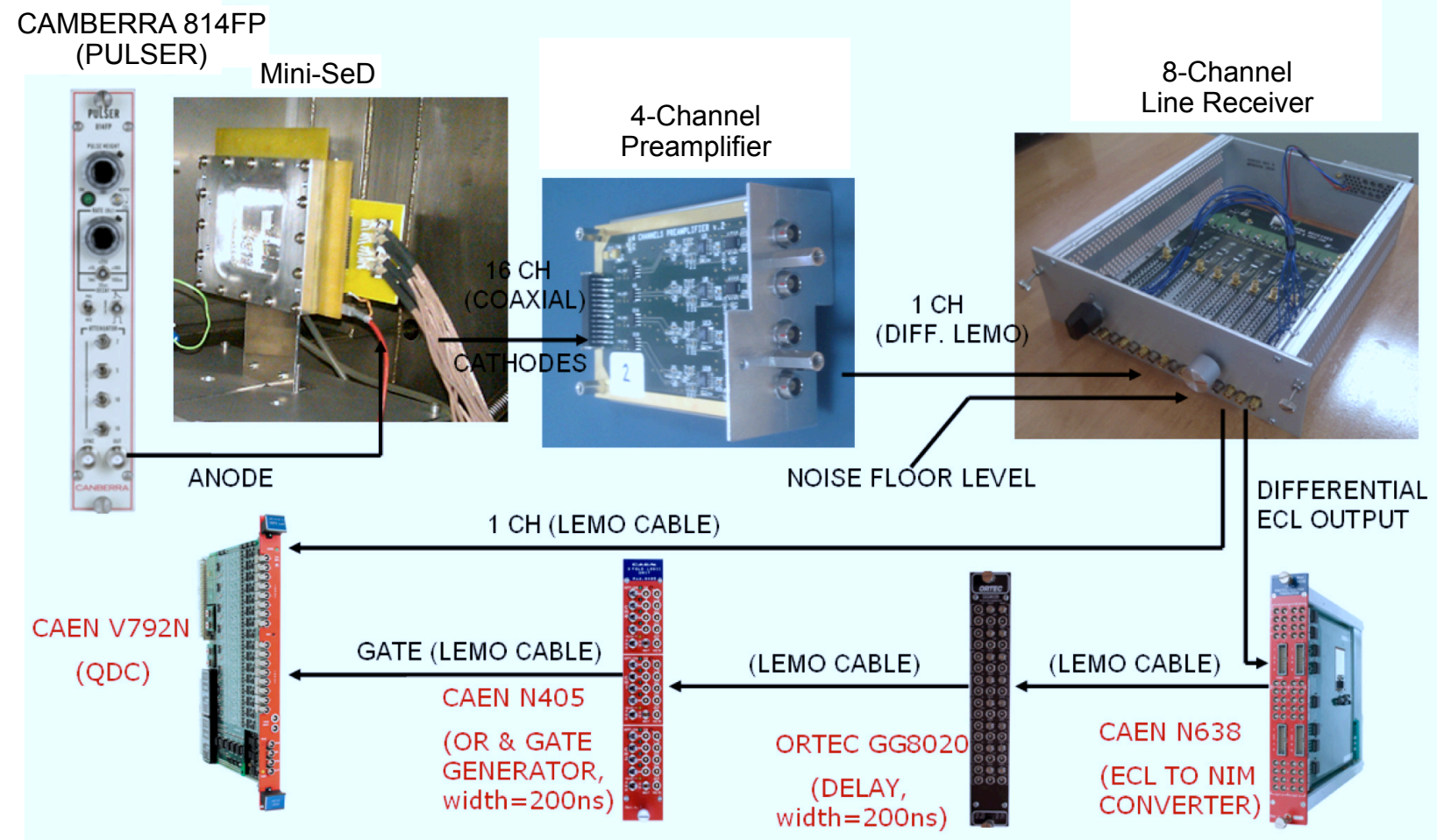

Fig. 9. Measurement set-up used in the pulse-generator experiment.
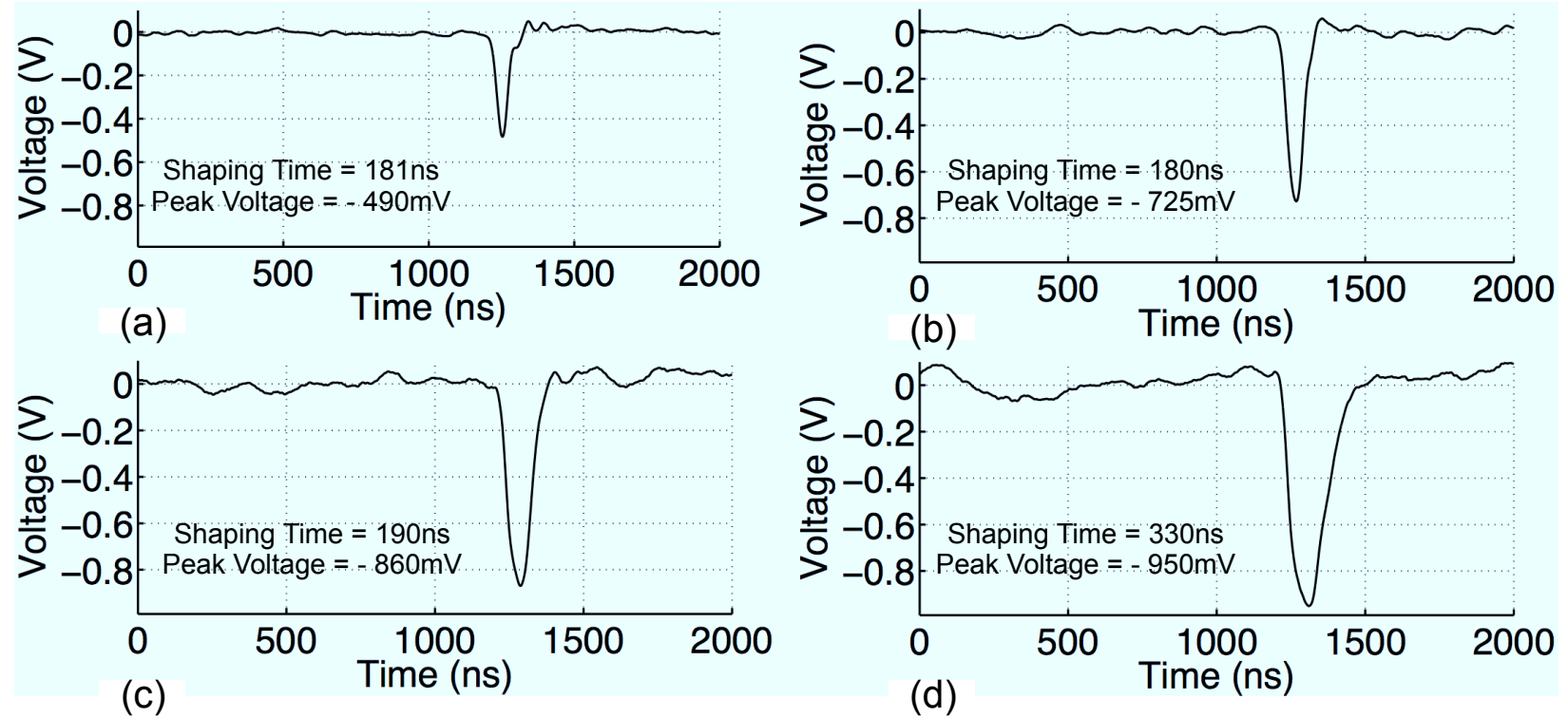

Fig. 10. Output waveforms of the line receiver for an input pulse and different values of the differential-to-single-ended gain. (a) Gain = 1, (b) Gain = 2, (c) Gain = 4, (d) Gain =8. 


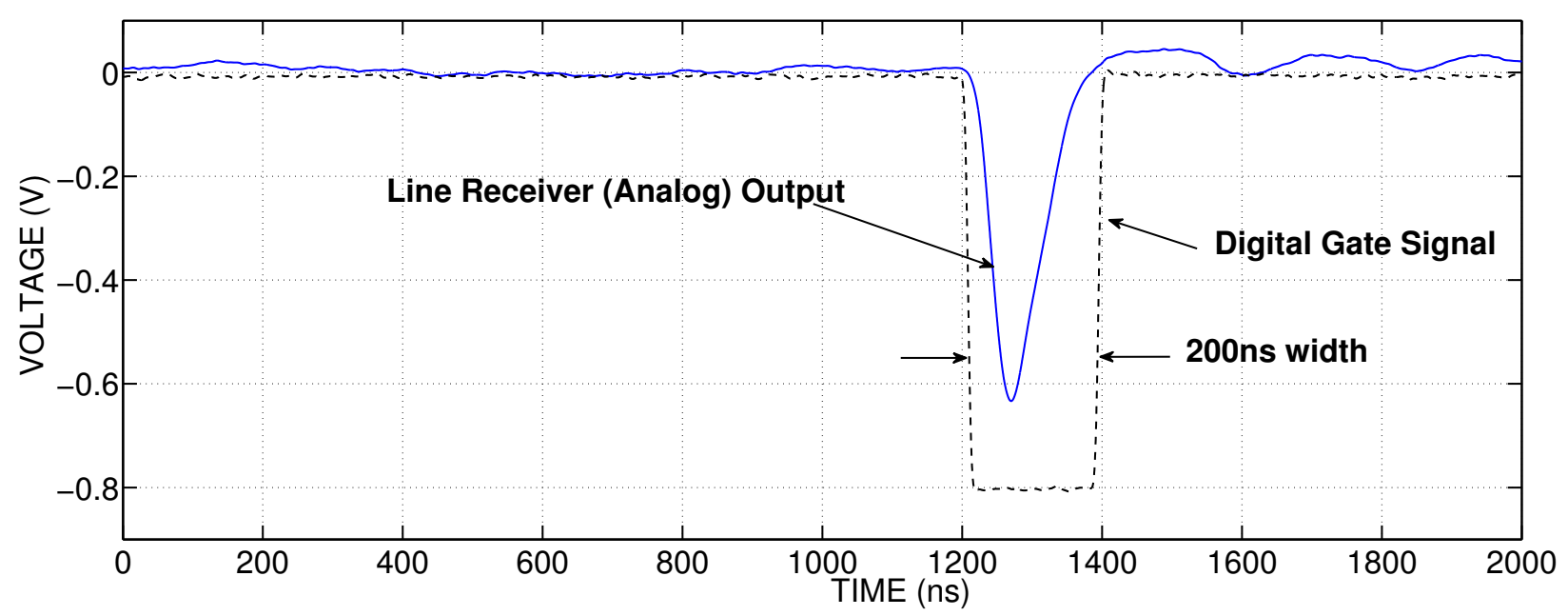

Fig. 11. QDC inputs: digital gate signal and line receiver analog output.

detected and integrated by the QDC, because the trigger signal is only generated when an input pulse is detected. The 200-ns width gate signal provided by this trigger is generated and delayed by the GG8020 module in order to be synchronised with the analog output, thus guaranteeing a correct detection of real events, even considering the effect of variations in the component values of different channels.

\section{B. Experiment with a Source of $\alpha$ Particles}

The second experiment was carried out using a triple source of Plutonium $\left({ }^{239} \mathrm{Pu}\right)$, Americium $\left({ }^{241} \mathrm{Am}\right)$ and Curium $\left({ }^{244} \mathrm{Cm}\right)$, which emit $\alpha$ particles with an average energy of $1.29 \mathrm{MeV} / \mathrm{u}$, $1.37 \mathrm{MeV} / \mathrm{u}$ and $1.48 \mathrm{MeV} / \mathrm{u}$, respectively. As illustrated in the instrumentation set-up shown in Fig. 8, the secondary electrons generated by the $\alpha$ particles in the emissive foil, are guided to the active volume of the detector, where they ionize the gas inside, generating electron-ion pairs. The produced electrons generate new ionizations, in the gas, which means more charge (ion and electrons) that are measured by the anode and cathodes and transmitted to the readout system. The electrons distribution around the cathodes gives us the position of the incident particle.

Fig. 12 shows the signal waveforms of the most important nodes of the readout system, corresponding to different gains of the line receiver, illustrating how the proposed readout system is able to process valid events obtained from real sources. Noise-level measurements are shown in the figure, demonstrating that a maximum peak SNR of $25.1 \mathrm{~dB}$ with shaping times of 170-230ns 
can be achieved.

\section{Experiment with a ${ }^{58} \mathrm{Ni}$ Particle Beam}

The third experiment was carried out using a Nickel $\left({ }^{58} \mathrm{Ni}\right)$ beam at a high counting rate, whose accelerated particles pass through an emissive foil, and as a result of the interactions, secondary electrons are generated and detected by the Mini-SeD (in the same way as explained in Section IV.B). In this case, a single channel silicon Surface Barrier Detector (SBD) is used to measure the particles of the ${ }^{58} \mathrm{Ni}$ beam after crossing the emissive foil (see Fig. 8). This event is also used by the trigger system generator to activate the QDC only when a real particle is detected.

Fig. 13 shows the main output waveforms obtained by the readout system, demonstrating a correct performance to process two consecutive events, with a time delay between them of approximately 400ns, which means a detection rate over $2.5 \cdot 10^{6} \mathrm{pps}$. Finally, Table VI shows the measured performance summary of the readout system, for the different experimental conditions considered. Note that the values of the measured SNR are approximately 5-10dB lower than those obtained by simulation - shown in Table V. This is mainly due to external noise sources and electromagnetic interferences, which are present in the measurement set-up and lab equipment. In order to attenuate these effects, different strategies were taken into account in the designed PCBs, including among others: the use of separate planes for digital and analog signals; regulators to keep the values of voltage supplies stable; decoupling capacitors in the supply, biasing and reference signals; ESD protection diodes, etc. However, in spite of applying these circuit techniques, it is not possible to completely remove some external interferences, especially in a complex lab environment like that considered in this work. Nevertheless, the values of the measured shaping times and peak SNRs achieved, demonstrate the suitability of the presented readout system to detect particles at rates over $10^{6} \mathrm{pps}$.

\section{CONCLUSiON}

A complete readout electronic system for precise spatial detection in tracking of low-energy ion beams at a rate over $10^{6} \mathrm{pps}$ has been presented. Instead of using a charge-sensitive preamplifier, the proposed system uses a multi-channel TIA circuit, which has demonstrated to be more efficient than previously reported preamplifiers to detect consecutive events with a delay below 


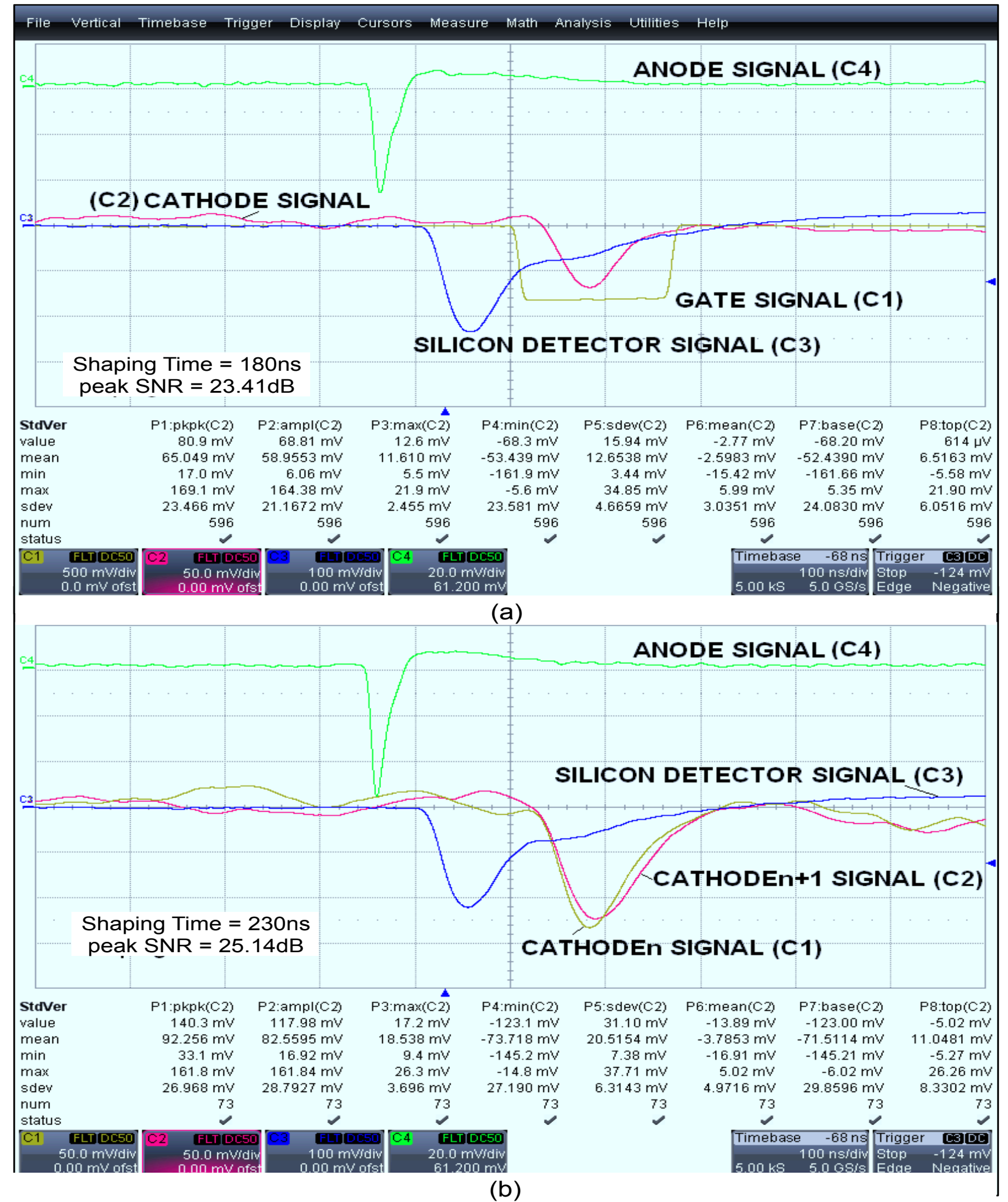

Fig. 12. Output waveforms of the readout system for an input source of $\alpha$ particles and different gains of the receiver line. (a) Gain $=2$. (b) Gain $=4$. 


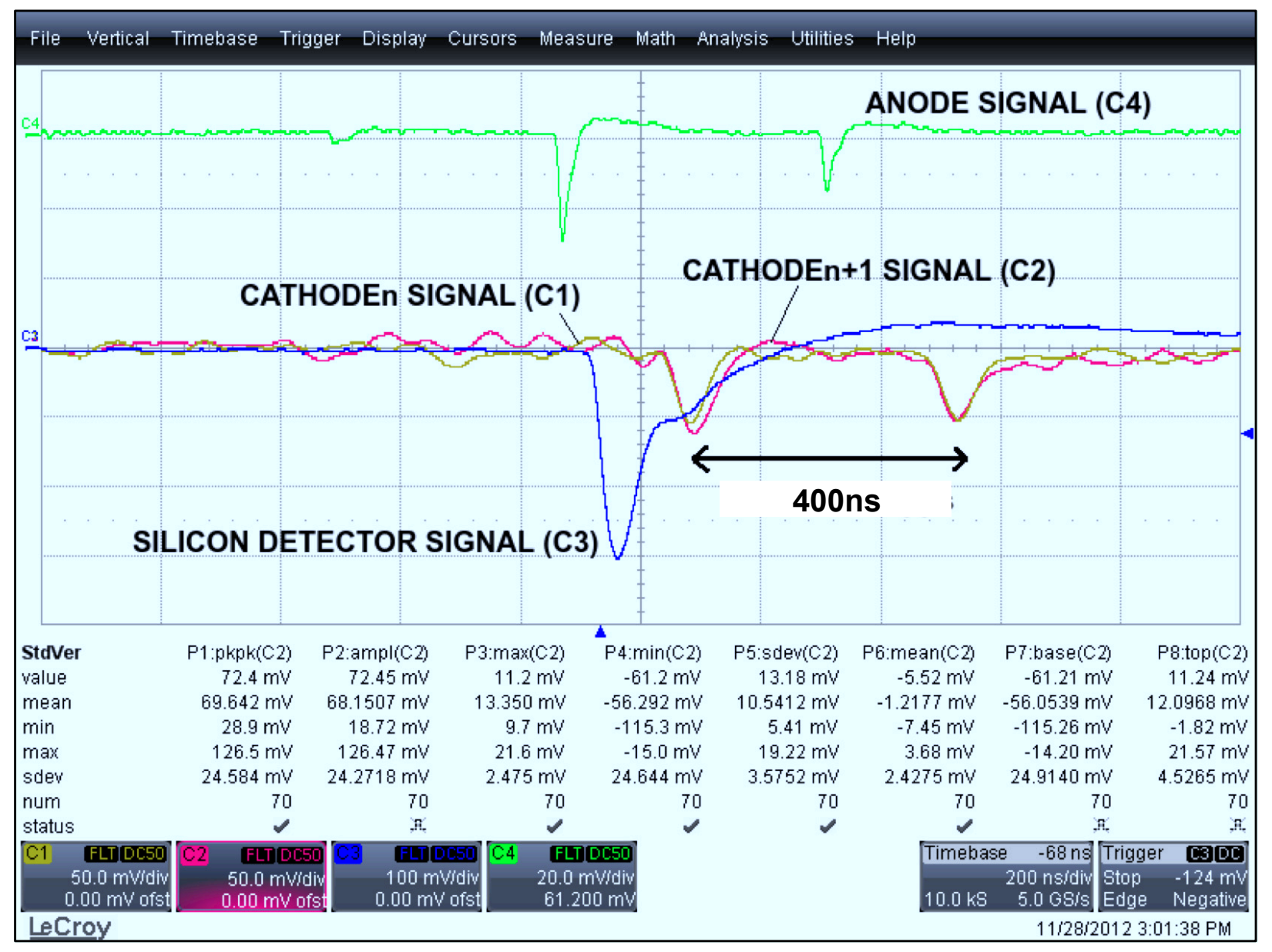

Fig. 13. Output waveforms of the readout system for a ${ }^{58} \mathrm{Ni}$ particle beam.

TABLE VI

MEASURED PERFORMANCE SUMMARY

\begin{tabular}{|c|c|c|}
\hline Receiver Gain & peak-SNR (dB) & Shaping Time (ns) \\
\hline \multicolumn{3}{|c|}{ Source of $\alpha$ particles } \\
\hline 1 & 23.2 & 170 \\
\hline 2 & 23.4 & 180 \\
\hline 4 & 25.1 & 230 \\
\hline \multicolumn{3}{|c|}{${ }^{58}$ Ni Particle Beam } \\
\hline 1 & 23.8 & 170 \\
\hline 2 & 23.8 & 180 \\
\hline 4 & 24.1 & 230 \\
\hline
\end{tabular}


400ns. The proposed front-end electronics is complemented by a line receiver to adapt the detected signals before being digitized by a QDC. These blocks are combined with other off-the-shelf electronic parts in order to target the required physical characteristics of MiniSecondary electron detectors. Diverse experimental measurements are shown to validate the presented approach, which constitutes a very promising strategy for the implementation of frontend electronic systems in the next generation of particle accelerators.

\section{ACKNOWLEDGMENT}

The authors would like to thank Miguel A. Cortés Giraldo for his help with GEANT4 simulations, and J. Pancin, E. Delagnes, and their colleagues at SEDI-IRFU Department at CEA-Saclay, for their support and assistance with some measurements.

\section{REFERENCES}

[1] J. K. Tuli, Nuclear Wallet Cards. National Nuclear Data Center, 2005.

[2] E. Bougamont et al., "A large tracking detector for low-energy ions," Nuclear Instruments and Methods in Physics Research, vol. 518, pp. 129-131, February 2004.

[3] J. Pancin et al., "Secondary electrons detectors for beam tracking: micromegas and wire chamber," Journal of Instrumentation, vol. 4, December 2009.

[4] E. Delagnes et al., "SFE16, a Low Noise Front-End Integrated Circuit Dedicated to the Read-out of Large Micromegas Detectors," IEEE Trans. on Nuclear Science, vol. 47, pp. 1447-1453, April 2000.

[5] A. Paul et al., "Accurate Nuclear Transition Data Determined by Prompt (n, $\gamma$ )-Spectrometry," IEEE Trans. on Instrumentation and Measurement, vol. 50, pp. 576-579, April 2001.

[6] H. P. Lima et al., "A Fast Multichannel-Analyzer for Radiation Detection Applications," IEEE Trans. on Instrumentation and Measurement, vol. 53, pp. 378-383, April 2004.

[7] L. Hervas et al., "The ATLAS Liquid Argon Electromagnetic Calorimeter: Construction, Comissioning and Selected Test Beam Results," IEEE Trans. on Instrumentation and Measurement, vol. 54, pp. 1505-1512, August 2005.

[8] F. Lugiez et al., "IDeF-X V1.1: Performances of a New CMOS 16 Channels Analogue Readout ASIC for Cd(Zn)Te Detectors," Proc. of 2006 IEEE Nuclear Science Symposium Conference Record, pp. 841-844, 2006.

[9] H. Spieler, "Front-end electronics and trigger systems - Status and Challenges," Nuclear Instruments and Methods in Physics Research, vol. 581, pp. 65-79, October 2007.

[10] P. Baron et al., "AFTER, an ASIC for the readout of the large T2K time projection chambers," IEEE Trans. on Nuclear Science, vol. 55, pp. 1865-1872, June 2008.

[11] M. Perenzoni et al., "A Multispectral Analog Photon-Counting Readout Circuit for X-ray Hybrid Pixel Detectors," IEEE Trans. on Instrumentation and Measurement, vol. 57, pp. 1438-1444, July 2008.

[12] M. Bolic, V. Drndarevic, and W. Gueaieb, "Pileup Correction Algorithms for Very-High-Count-Rate Gamma-Ray Spectrometry With $\mathrm{NaI}(\mathrm{Tl})$ Detectors," IEEE Trans. on Instrumentation and Measurement, vol. 59, pp. 122-130, January 2010. 
[13] S. Corbellini et al., "Modified POF Sensor for Gaseous Hydrogen Fluoride Monitoring in the Presence of Ionizing Radiations," IEEE Trans. on Instrumentation and Measurement, vol. 61, pp. 1201-1208, May 2012.

[14] S. Zimmermann et al., "Implementation and Performance of the Electronics and Computing System of the Gamma Ray Energy Tracking In-Beam Nuclear Array (CRETINA)," IEEE Trans. on Nuclear Science, vol. 59, pp. 2494-2500, October 2012.

[15] G. Balbi et al., "Electron Interference via a 4096-Pixel MAPS Detector Designed for High-Energy Physics Experiments," IEEE Trans. on Nuclear Science, vol. 60, pp. 913-917, April 2013.

[16] Y. Kong et al., "A Prototype Compton Camera Array for Localization and Identification of Remote Radiation Sources," IEEE Trans. on Nuclear Science, vol. 60, pp. 1066-1071, April 2013.

[17] E. G. Bakhoum, M. H. Cheng, and K. M. V. Landingham, “Alpha-Particle-Based Icing Detector for Airchaft," IEEE Trans. on Instrumentation and Measurement, vol. 63, pp. 185-191, January 2014.

[18] H. Spieler, Semiconductor Detector Systems. Oxford University Press, 2005.

[19] R. Adler et al., "The CPLEAR detector at CERN," Nuclear Instruments and Methods in Physics Research, vol. 379, pp. 76-100, September 1996.

[20] V. Spanoudaki et al., "An analog signal processing ASIC for a small animal LSOAPD PET tomograph," Nuclear Instruments and Methods in Physics Research, vol. 564, pp. 451-462, August 2006.

[21] A. Garzón-Camacho et al., "A Preamplifier for the Front-End Readout System of Particles Tracking in Secondary Electron Detectors," Proc. of IEEE International Symposium on Circuits and Systems (ISCAS), pp. 1171-1174, May 2012.

[22] A. Drouart et al., "A gas secondary electron detector," Nuclear Instruments and Methods in Physics Research, vol. 477, pp. 401-405, January 2002.

[23] S. Agostinelli et al., "Geant4: A simulation toolkit," Nuclear Instruments and Methods in Physics Research, vol. 506, no. 3, pp. 250-303, 2003.

[24] J. Allison et al., "Geant4 Developments and Applications," IEEE Trans. on Nuclear Science, vol. 53, pp. 270-278, February 2006.

[25] F. Bal et al., "The Readout System of the CPLEAR Electromagnetic Calorimeter," Nuclear Instruments and Methods in Physics Research, vol. 323, pp. 511-518, December 1992.

[26] MIDAS: Multi Instance Data Acquisition System. [Online]. Available: http://npsg.dl.ac.uk/MIDAS., 2013.

[27] Multisim ${ }^{\mathrm{TM}}$ User Guide. National Instruments, 2013.

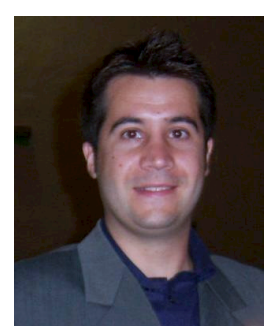

Alejandro Garzón-Camacho received the B.S. degree in 2004 in Telecommunication Engineering, the B.S. degree in Electronics Engineering in 2010 and the M.S. degree in Microelectronics in 2013, all of them from the University of Seville, Seville, Spain. He is currently working toward the Ph.D. degree in Physical Sciences and Technologies at University of Seville, Seville, Spain.

He was working from 2009 to 2013 at the National Center of Accelerators of Seville (CNA, CSIC/ University of Seville), where he was with the Basic Nuclear Physics Research Unit (FNB). He is currently working as a researcher at the Institute for the Structure of Matter (IEM, CSIC) in Madrid, Spain. His main areas of interest are in the field of analog and digital fast readout electronic systems for nuclear particle detectors. 


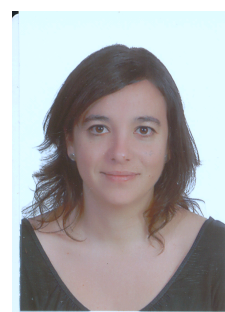

Begoña Fernández received her M.S degree in physics in 2006 from the University of Seville and her PhD in Nuclear Physics in 2014. Since 2007, she is working at the National Accelerator Center (CNA) in Seville, a joint institute that belongs to the University of Seville, CSIC and "Junta de Andalucía". There, she is the person in charge of the Nuclear Physics Line at the Tandem of $3 \mathrm{MV}$ and of the detectors laboratory.

During the last years she was working in the research and development of low pressure gaseous detectors for beam tracking in collaboration with the CEA-Saclay and GANIL (France). Her experience is based on Experimental Nuclear Physics and detectors.

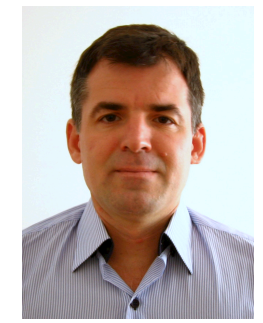

Marcos A. G. Alvarez graduated in Physics, during the period 1992-1996 and post-graduated, with a Master thesis in Science (1996-1998) and his PhD in Nuclear Physics (1999-2002). All these stages were developed in the Physics Institute of the University of São Paulo (IFUSP), in Brazil. His first post-doctorate was carried out at "Commissariat a l' Energie Atomique" (CEA) in Saclay, France (2003-2004). In 2004, Dr. M. A. G. Alvarez was contracted as Superior Title Researcher by the University of Seville (USE), in Spain (2004-2007), following in the same university, as "Researcher Doctor", during the period 20082013, through the programs "Juan De La Cierva" and "Ramon y Cajal", respectively. During his stay at the University of Seville, he was accredited as a Full Professor by the Spanish National Agency for Quality Assessment and Accreditation (ANECA). In 2013, still in the University of Seville, he worked as visiting professor at the University of São Paulo, funded by the "Research Foundation of São Paulo State" (FAPESP). In the same year, he was approved in a public process, to be a "contracted Doctor" of the Physics Institute of the University of São Paulo (IFUSP), which is his current permanent position.

His experience is based on Nuclear Physics, mainly on nuclear reactions and instrumentation, acting in the development of detectors and electronics, besides nuclear reaction experimental measurements involving stable and exotic nuclei, with the application of both research lines in Medical Physics. Such experience was achieved with several experimental campaigns carried out at the main international laboratories (CERN, GANIL, GSI, TRIUMF, IPN-Orsay, among others) and the participation in several international research projects.

Dr. Alvarez took part into several academic projects, creating and teaching different courses for graduation and post-graduation levels. He supervised 3 doctoral theses, 5 master-degree research works and 1 post-doctoral stage. As a result of his research activity, Dr. M. A. G. Alvarez has published more than 80 international reviews, achieving approximately 1000 citations. Dr. M. A. G. Alvarez is reviewer of the "Physical Review Journals" and "Reviews of Modern Physics" of "American Physics Society (APS)". 


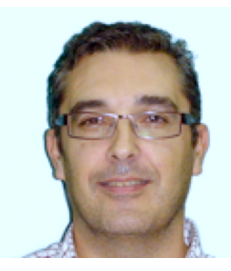

Joaquín Ceballos received the M.S. degree in Physics in 1994 from the University of Seville, Spain. Since 1997 he has been working at the Institute of Microelectronics of Seville (IMSE), which is in turn part of the Spanish Microelectronics Center (CNM) of the Spanish National Research Council (CSIC), where he has participated in numerous research projects funded by the EU, national programs and companies.

Since 2005 he is in charge of test coordination at IMSE and in 2013 became Technical Vice-director of IMSE. Since 2007 he has taken part in activities related to design of ASICs for space and characterization of rad-hard technologies. He also collaborates with National Center of Accelerators of Seville (CNA, CSIC/University of Seville) in some projects related to electronic detectors for particle tracking.

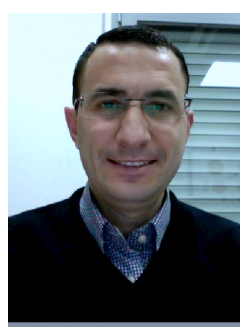

José M. de la Rosa (S'96-M'01-SM'06) received the M.S. degree in Physics in 1993 and the Ph.D. degree in Microelectronics in 2000, both from the University of Seville, Spain. Since 1993 he has been working at the Institute of Microelectronics of Seville (IMSE), which is in turn part of the Spanish Microelectronics Center (CNM) of the Spanish National Research Council (CSIC). He is also with the Department of Electronics and Electromagnetism of the University of Seville, where he is currently an Associate Professor, accredited as a Full Professor.

His main research interests are in the field of analog and mixed-signal integrated circuits, especially high-performance data converters, including analysis, behavioral modeling, design and design automation of such circuits. In these topics, Dr. de la Rosa has participated in a number of National and European research and industrial projects, and has co-authored some 200 international peer-reviewed publications, including journal and conference papers, 10 book chapters and 5 books.

Dr. de la Rosa is a Member-at-Large of the IEEE-Spain Section and a member of the Analog Signal Processing Technical Committee of the IEEE Circuits and Systems Society, where he serves as the Secretary of the Spanish Chapter. He serves as Associate Editor for IEEE Transactions on Circuits and Systems I: Regular Papers, where he received the 2012-2013 Best Associate Editor Award. He is also a member of the Steering Committee of IEEE MWSCAS conference and he serves in the technical committees of diverse IEEE conferences, including ISCAS, MWSCAS, ICECS, LASCAS, VLSI-SoC and DATE, being TPC co-chair of MWSCAS 2012 and ICECS 2012, and recently appointed as TPC co-chair of IEEE LASCAS 2015. 